EDITORIAL

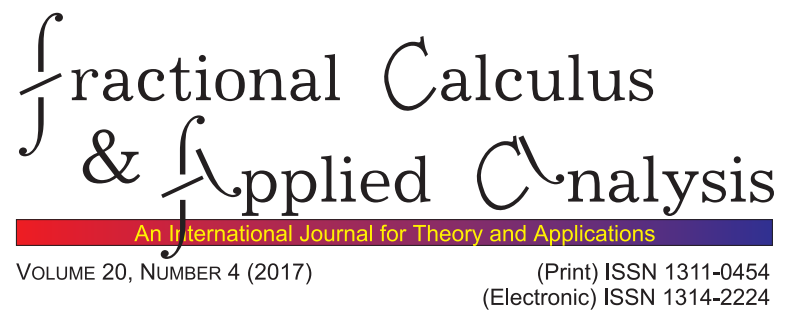

\title{
FCAA RELATED NEWS, EVENTS AND BOOKS
} (FCAA-VOLUME 20-4-2017)

Dear readers,

\section{Virginia Kiryakova}

in the Editorial Notes we announce news for our journal, anniversaries, information on international meetings, events, new books, etc. related to the FCAA ("Fractional Calculus and Applied Analysis") areas.

\section{Reviews on New Books}

Yong Zhou, JinRong Wang, Lu Zhang, Basic Theory of Fractional Differential Equations. 2nd Ed. World Scientific, London (2016), 380 pp., ISBN: 978-981-3148-16-1 (hardcover), 978-981-3148-18-5 (ebook). Details: http://www.worldscientific.com/worldscibooks/10.1142/10238.

Review on this book: This monograph is devoted to a rapidly developing area of research: the qualitative theory of fractional differential equations. It provides the necessary background material required to go further into the subject and explore the rich research literature. The contents are very recent and comprehensive, based on the research carried out by the authors and other experts during the past seven years. In this edition, two new topics have been added: fractional impulsive differential equations, and time-fractional partial differential equations including fractional-order generalizations of the diffusion, Navier-Stokes, Schrödinger and Euler-Lagrange equations.

After introducing some facts from fractional calculus, nonlinear analysis and semigroup theory, various types of fractional differential equations are studied. The most attention is paid to nonlinear equations, including discontinuous fractional functional differential equations with bounded, unbounded and infinite delay, respectively, fractional differential equations in Banach spaces, fractional impulsive differential equations, and timefractional partial differential equations. The tools used in the monograph include classical and modern nonlinear analysis methods such as fixed point theory, measure of noncompactness method, topological degree method, the technique of Picard operators, and critical point theory. The classical semigroup theory and the subordination principle for the linear abstract Cauchy problem of fractional order are also employed.

(c) 2017 Diogenes Co., Sofia

pp. 825-828, DOI: $10.1515 /$ fca-2017-0043

DE GRUYTER 
The monograph is well written, and every chapter is equipped with an interesting introduction. The theory of fractional ordinary and partial differential equations developed in the book is very useful for readers who want to do theoretical work in this area. It can serve as well as a starting point for further research concerning dynamics, control, numerical analysis and applications of fractional differential equations.

Contents: (7 chapters)

- Preliminaries

- Fractional Functional Differential Equations

- Fractional Ordinary Differential Equations in Banach Spaces

- Fractional Abstract Evolution Equations

- Fractional Impulsive Differential Equations

- Fractional Boundary Value Problems

- Fractional Partial Differential Equations

Sample Chapter: Chapter 1: Preliminaries (available at the website)

Readership: Researchers and graduate or $\mathrm{PhD}$ students dealing with fractional calculus and applied analysis, differential equations and related areas of research.

Reviewer: Emilia Bazhlekova

Karabi Biswas, Gary Bohannan, Riccardo Caponetto, António Lopes, J. A. Tenreiro Machado, Fractional Order Devices. Springer, SpringerBriefs in Nonlinear Circuits (2017), 110 pp. + xi + 79 illustr., ISBN: 978-3-319-54459-5 (hardcover), 978-3-319-54460-1 (e-book).

Details: http://www.springer.com/gp/book/9783319544595.

\section{Features:}

- Provides readers with new tools for designing new types of integrated circuits

- Shows how fractional-order controllers can be given practical implementation

- Increases readers understanding of how ostensibly unrelated systems can contribute to circuit design

Annotation: This book focuses on two specific areas related to fractional order systems the realization of physical devices characterized by noninteger order impedance, usually called fractional-order elements (FOEs); and the characterization of vegetable tissues via electrical impedance spectroscopy (EIS) and provides readers with new tools for designing new types of integrated circuits. The majority of the book addresses FOEs.

The interest in these topics is related to the need to produce "analogue" electronic devices characterized by non-integer order impedance, and to the 
characterization of natural phenomena, which are systems with memory or aftereffects and for which the fractional-order calculus tool is the ideal choice for analysis.

FOEs represent the building blocks for designing and realizing analogue integrated electronic circuits, which the authors believe hold the potential for a wealth of mass-market applications. The freedom to choose either an integer- or non-integer-order analogue integrator/derivator is a new one for electronic circuit designers. The book shows how specific non-integer-order impedance elements can be created using materials with specific structural properties.

EIS measures the electrical impedance of a specimen across a given range of frequencies, producing a spectrum that represents the variation of the impedance versus frequency a technique that has the advantage of avoiding aggressive examinations.

Biological tissues are complex systems characterized by dynamic processes that occur at different lengths and time scales; this book proposes a model for vegetable tissues that describes the behavior of such materials by considering the interactions among various relaxing phenomena and memory effects.

Contents: (5 chapters)

- Introduction to Fractional Order Elements and Devices

- Devices

- Demonstrations and Applications of Fractional Order Devices

- Fractional-order Models of Vegetable Tissues

- Future Directions

\section{Call for chapters in new volume "Fractional Calculus for Complex Systems" in Encyclopedia of Complexity and Systems Science (2nd Ed.)}

This volume in the "Encyclopedia of Complexity and Systems Science, Second Edition", offers a detailed account of fractional calculus tools, signatures of complex systems, hidden connections to fractional calculus, and applications and case studies involving fractional calculus in complex signal analysis and complex system modelling, analysis and control (MAD). The authors document both the foundational concepts of fractional calculus in complexity science as well as their applications to, and role in the optimization of, complex engineered systems. Fractional calculus is about differentiation and integration of non-integer orders. Convenience has driven the use of integer-order models and controllers for complex natural or man-made systems, but these traditional models and tools for the control of dynamic systems may result in suboptimum performance and 
even "anomalous" phenomena. In contrast, the growing literature documents "more optimal" performance when fractional order calculus tools are applied. From an engineering point of view, new and beneficial uses of this versatile mathematical tool are both possible and important, and may become an enabler of new science discoveries.

- Presents the first comprehensive coverage of the fractional calculus role in complex systems.

- Discusses major existing signatures such as power law of complex systems with emphasis on the connections to the fractional calculus

- Includes a wide variety of real world case studies in signal analysis and complex system modeling and control

Topic Areas (Table of Contents in preparation):

- Fractional calculus:

- Definitions, history, basic properties; - Fractional order dynamic systems; - Fractional noises

- Signatures of complex systems and its fractional calculus connection:

- Power law; - Long range dependence; - Long memory; - Long range interaction; - Heavy tailedness; - etc.

- Complex signal analysis using fractional calculus

- Complex system modeling using fractional calculus

- Complex system control using fractional calculus

Key parameters: Minimum number of chapters 30 to 50 (no upper limit); 9,000-12,000 words (plus figures and references) per chapter (or 10-12 published pages); delivery date 12/31/2018; submission via online system, only at https://meteor.springer.com.

If you are interested in contributing a chapter in this new volume "Fractional Calculus for Complex Systems", please visit the website

http://mechatronics.ucmerced.edu/fccs

for Guidelines and background information. Send me a chapter proposal (one page) with title/authors/affiliations/contact info/synopsis/keywords with FCCS on email, subject: title for easy search. Thank you!

YangQuan Chen, e-mail: yqchen@ieee.org

June/24/2017

Virginia Kiryakova, Institute of Mathematics and Informatics

Bulgarian Academy of Sciences, Acad. G. Bontchev Str., Block 8

Sofia1113-BULGARIA, e-mail:virginia@diogenes.bg

Please cite to this paper as "Ed. Note, FCAA-Volume 20-4-2017", publ. in: Fract. Calc. Appl. Anal., Vol. 20, No 4 (2017), pp. 825-828, DOI: $10.1515 /$ fca-2017-0043 